\title{
Analysis of Signal Processing Techniques for Optical 112 Gb/s DP-QPSK Receivers with Experimental Data
}

\author{
Thiago F. Portela, Diego V. Souto, Valery N. Rozental, Hugo B. Ferreira, Darli A. A. Mello \\ OCNLab, Department of Electrical Engineering, University of Brasilia \\ Helmut Griesser ${ }^{1}$ \\ Advanced Technology, Ericsson
}

\begin{abstract}
This paper evaluates several signal processing techniques for optical dual-polarization quadrature phase-shift keying (DP-QPSK) coherent receivers, using offline post-processing of experimental data. In addition to electronically separating the two polarization multiplexed signals, the investigated signal processing algorithms also compensate for chromatic dispersion (CD) and polarization mode dispersion (PMD). In particular, we investigate two distinct architectures:

1) Constant modulus algorithm (CMA) equalization followed by feedforward phase recovery.

2) Decision-directed least mean square (DD-LMS) equalization combined with decision-directed phase recovery.

The interplay of both architectures with bulk dispersion compensators and carrier recovery algorithms is also analyzed.
\end{abstract}

Index Terms - Blind equalization, coherent receiver, optical communications, polarizations demultiplexing.

\section{INTRODUCTION}

The increasing demand for data traffic in optical networks has been pushing the development of spectrally-efficient systems using polarization multiplexing and multilevel modulation formats. In this context, dual-polarization quadrature phase-shift keying (DP-QPSK) transmission emerged as an attractive alternative. Such systems convey four bits per symbol (considering both polarization orientations), consequently reducing the symbol rate by the same factor in comparison to a binary system at the same bit rate. In addition to relaxing hardware requirements, the reduced symbol rate also accounts for an increased tolerance to inter-symbol interference (ISI). If coherently detected, polarization multiplexed QPSK signals can be separated at the receiver by signal processing algorithms, and linear effects, such as chromatic dispersion (CD) and polarization mode dispersion (PMD), can be compensated for standard equalization techniques. Indeed, DP-QPSK with coherent detection has been considered a viable alternative for future $100 \mathrm{GbE}$ transmission (at a data rate of $112 \mathrm{~Gb} / \mathrm{s}$, or $28 \mathrm{Gbaud}$ ). While real-time $100 \mathrm{GbE}$ transceivers are not yet commercially available, research on the signal processing algorithms for the receiver has been relying on the offline postprocessing of experimentally captured data, using fast oscilloscopes.

\footnotetext{
${ }^{1}$ now with ADVA optical networking. 
This paper evaluates several signal processing techniques for optical dual-polarization quadrature phase-shift keying (DP-QPSK) coherent receivers, using offline post-processing. In particular, we investigate two distinct architectures: constant modulus algorithm (CMA) equalization followed by feedforward phase recovery; and decision-directed least mean square algorithm (DD-LMS) equalization combined with decision-directed phase recovery. The interplay of both architectures with bulk dispersion compensators and frequency recovery algorithms is also analyzed. The paper is structured as follows: Section 2 describes the signal processing algorithms investigated in the paper; Section 3 presents the experimental results obtained by offline post-processing; lastly, Section 4 concludes the paper.

\section{SIGNAL PROCESSING ALGORITHMS}

\section{A. Resampling, Normalization and Orthogonalization}

Figure 1 shows the canonic chain of signal processing algorithms commonly employed in DPQPSK receivers. The first block is an experimental artifice used to adapt the sampling rate of fast oscilloscopes to the data rate of the consecutive signal processing algorithms. The subsequent orthogonalization and normalization block counteracts eventual quadrature imbalances generated at the receiver front-end [1], and is followed by bulk dispersion compensators. Residual CD and PMD are then compensated in the butterfly adaptive equalizer. Lastly, phase noise effects are mitigated in the phase recovery module. An additional frequency recovery algorithm may be placed in several positions, and therefore was omitted from the figure.

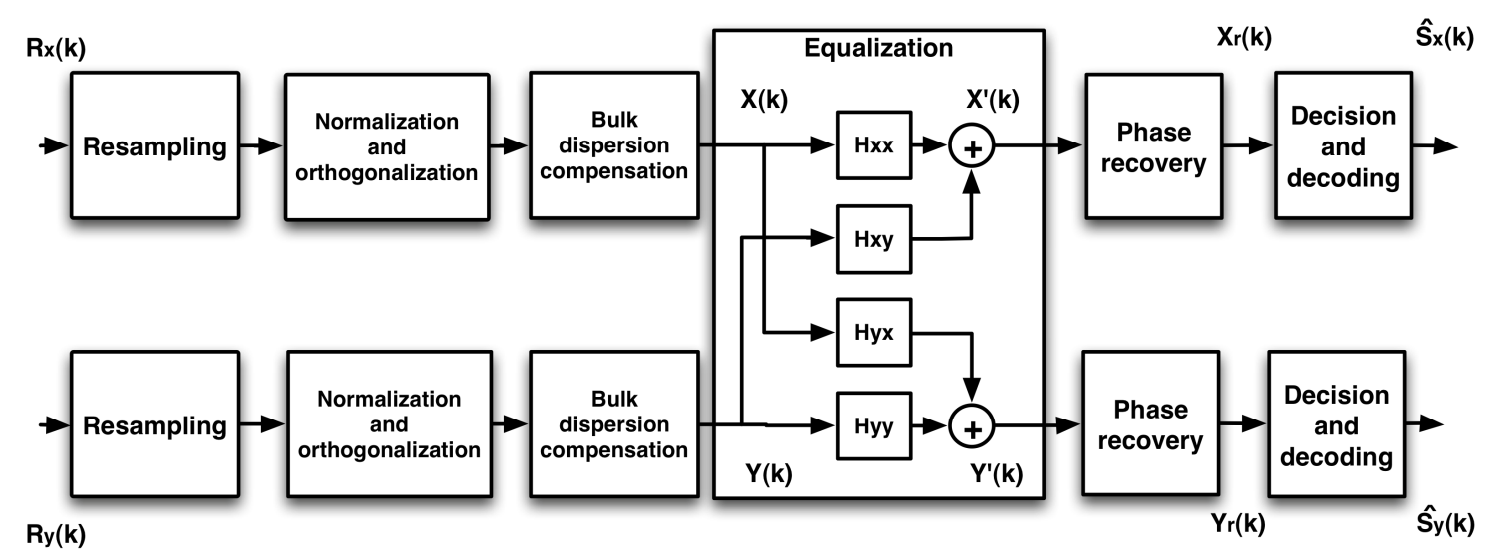

Fig. 1. Chain of signal processing algorithms for a coherent DP-QPSK receiver.

In this work, the system operates at 28 Gbaud, and data is sampled at 50 GSamples/s. Since equalization is accomplished by $T_{s} / 2$-spaced equalizers, the input data is resampled at a $56 / 50$ rate.

\section{B. Bulk Dispersion Compensation}

Dispersion compensation in optical coherent receivers may be carried out by separate blocks: a bulk equalizer with fix coefficients that compensates for most of the $\mathrm{CD}$ accumulated on the link, and an adaptive equalizer that compensates for any residual dispersion. In particular, the time-domain algorithm investigated in this paper [2] attempts to perform zero-forcing equalization, which may be 
an interesting alternative given the flat amplitude response of the $\mathrm{CD}$ transfer function. The coefficients $a_{k}$ of the resulting finite impulse response (FIR) filter are given by:

$$
\begin{gathered}
a_{k}=\sqrt{\frac{-j c T^{2}}{D \lambda^{2} z}} e^{j \frac{\pi c T^{2}}{D \lambda^{2} z} k^{2}} \\
-\left\lfloor\frac{N}{2}\right\rfloor \leq k \leq\left\lfloor\frac{N}{2}\right\rfloor \text { and } N=2\left\lfloor\frac{|D| \lambda^{2} z}{2 c T^{2}}\right\rfloor+1,
\end{gathered}
$$

where $D$ is the fiber dispersion coefficient, $\lambda$ is the operating wavelength, $z$ is the propagation distance, $c$ is the speed of light, and $T$ is the sampling time.

Figures 2 and 3 show the amplitude and phase responses of the time-domain compensator for $D=$ $17 \mathrm{ps} /[\mathrm{nm} \cdot \mathrm{km}], z=50 \mathrm{~km}$, and $T=1 /\left(56 \cdot 10^{9}\right) \mathrm{s}$ (corresponding to the total accumulated dispersion of the experimental setup). Although the amplitude response is relatively flat in the central part of the filter, the ripple around $15 \mathrm{GHz}$ may amplify noise components. As for the phase response, the group delay in the main portion of the filter exhibits a satisfactory agreement with the ideal curve.

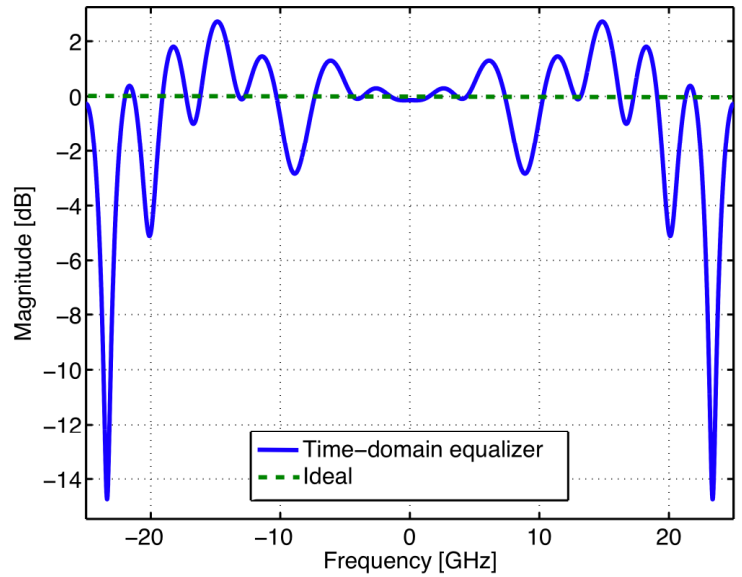

Fig. 2. Bulk dispersion compensating filters. Amplitude response.

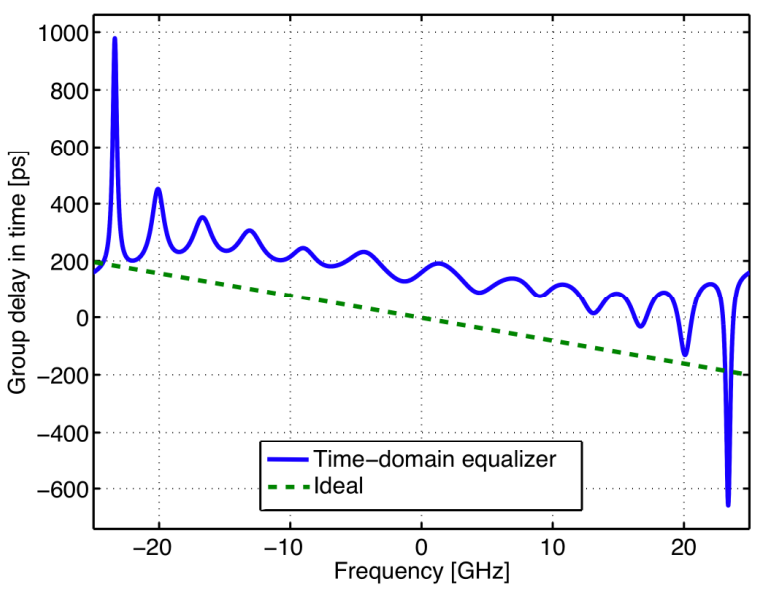

Fig. 3. Bulk dispersion compensating filters. Phase response.

\section{Butterfly Equalizer and Phase Recovery}

The adaptive butterfly equalizer separates the two polarization multiplexed signals and compensates for linear fiber effects such as PMD and residual CD that is left over from the bulk compensator.

The M-taps FIR filters $\boldsymbol{H}_{x x}, \boldsymbol{H}_{y x}, \boldsymbol{H}_{x y}$ and $\boldsymbol{H}_{y y}$ may be updated according to a stochastic gradient algorithm [2], [3]:

$$
\begin{aligned}
\boldsymbol{H}_{x x} & \rightarrow \boldsymbol{H}_{x x}+\mu \varepsilon_{x} \boldsymbol{X}(k)^{H}, \\
\boldsymbol{H}_{y x} & \rightarrow \boldsymbol{H}_{y x}+\mu \varepsilon_{x} \boldsymbol{Y}(k)^{H}, \\
\boldsymbol{H}_{x y} & \rightarrow \boldsymbol{H}_{x y}+\mu \varepsilon_{y} \boldsymbol{X}(k)^{H}, \\
\boldsymbol{H}_{y y} & \rightarrow \boldsymbol{H}_{y y}+\mu \varepsilon_{y} \boldsymbol{Y}(k)^{H},
\end{aligned}
$$

where $\mu$ is the step-size, $\varepsilon_{x}(k)$ and $\varepsilon_{y}(k)$ are the error signals that drive the equalization process. Terms $\boldsymbol{X}(k)^{H}$ and $\boldsymbol{Y}(k)^{H}$ are transpose and complex conjugates of $\boldsymbol{X}(k)=[X(k) X(k-1) \ldots X(k-$ $M+1)]^{T}$ and $\boldsymbol{Y}(k)=[Y(k) Y(k-1) \ldots Y(k-M+1)]^{T}$, respectively. 
The equalizer outputs $X^{\prime}(k)$ and $Y^{\prime}(k)$ are obtained as:

$$
\begin{aligned}
& X^{\prime}(k)=\boldsymbol{H}_{x x} \boldsymbol{X}(k)+\boldsymbol{H}_{y x} \boldsymbol{Y}(k), \\
& Y^{\prime}(k)=\boldsymbol{H}_{x y} \boldsymbol{X}(k)+\boldsymbol{H}_{y y} \boldsymbol{Y}(k) .
\end{aligned}
$$

The next sections particularize the error signal for the butterfly equalizers.

D. System 1

This system uses the constant-modulus algorithm (CMA) for signal equalization, so the error signal is computed as:

$$
\begin{aligned}
& \varepsilon_{x}(k+1)=\left(1-\left|X^{\prime}(k)\right|^{2}\right) X^{\prime}(k), \\
& \varepsilon_{y}(k+1)=\left(1-\left|Y^{\prime}(k)\right|^{2}\right) Y^{\prime}(k) .
\end{aligned}
$$

Since the CMA attempts to minimize variations of $\left|X^{\prime}(k)\right|$ and $\left|Y^{\prime}(k)\right|$ with respect to a constant value [4], [5], the equalization process is immune to phase-noise or frequency offsets. Accordingly, phase noise may be subsequently mitigated by the Viterbi \& Viterbi feedforward phase recovery algorithm, as depicted in Fig. 4.

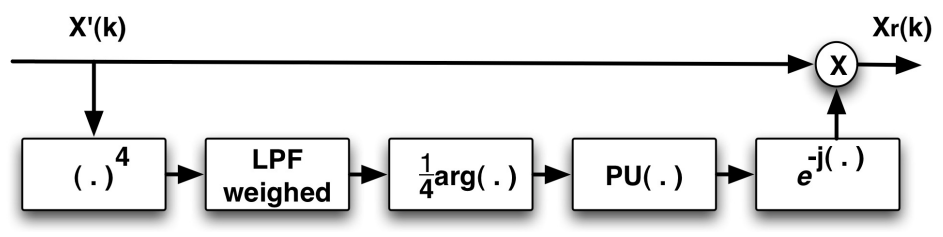

Fig. 4. The Viterbi \& Viterbi phase recovery algorithm.

The first step of the algorithm is to remove the data dependency by raising the complex signal to the fourth power. Afterwards, the sequence is filtered in order to minimize the influence of additive noise in the estimation process [6], and the signal argument is divided by four. The resulting argument sequence is then submitted to a phase unwrapper (PU) [7], to allow the resulting phase to vary between $-\infty$ to $+\infty$, instead of being limited between $-\frac{\pi}{4}$ and $+\frac{\pi}{4}$. Note that the filters corresponding to the " $X$ " and " $Y$ " outputs are updated independently, so singularities may occur (the same input being produced in both outputs). Several singularity-avoidance techniques have been recently proposed, exhibiting no penalty when compared to the standard CMA [8]. In this paper, we limited ourselves to verifying that the standard CMA equalizer converged to a singularity-free solution.

\section{E. System 2}

This system is based on a CMA startup followed by the decision-directed least mean square algorithm (DD-LMS) filter tracking, as shown in Fig. 5. The DD-LMS filter updating rule is given by [3]:

$$
\begin{aligned}
& \varepsilon_{x}(k+1)=e^{j \widehat{\theta}_{x}(k)}\left(\hat{S}_{x}(k)-X_{r}(k)\right), \\
& \varepsilon_{y}(k+1)=e^{j \widehat{\theta}_{y}(k)}\left(\hat{S}_{y}(k)-Y_{r}(k)\right),
\end{aligned}
$$

where $\hat{S}_{x}(k)$ and $\hat{S}_{y}(k)$ are the resulting symbols after minimum distance decision. Note in this case that the phase recovery process occurs along with the equalization process: 


$$
\begin{aligned}
& X_{r}(k)=X^{\prime}(k) e^{-j \widehat{\theta}_{x}(k),} \\
& Y_{r}(k)=Y^{\prime}(k) e^{-j \widehat{\theta}_{y}(k)},
\end{aligned}
$$

where the estimated phases $\hat{\theta}_{x}(k)$ and $\hat{\theta}_{y}(k)$ are given by:

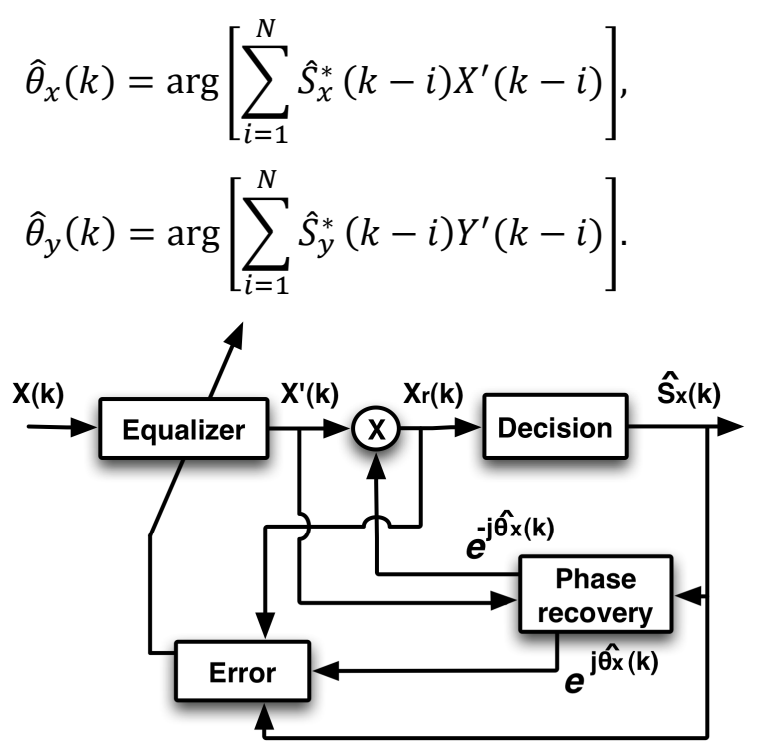

Fig. 5. DD-LMS equalization for the $X$ signal.

\section{F. Frequency Recovery}

Frequency recovery algorithms compensate for a possible frequency offset between transmitter and local oscillator lasers. Once this frequency offset $\Delta f$ is estimated, frequency recovery may be easily implemented by multiplying the equalized symbols $X^{\prime}(k)$ by the accumulated phase-shift $P S(k)=$ $e^{-j 2 \pi \Delta f T_{S} k}=P S(1)^{k}$, where $T_{S}$ is the symbol period. In this paper, we analyze two frequency recovery algorithms:

- The phase-difference algorithm estimates the phase-shift $P S(1)$ from the equalized samples $X^{\prime}(k)$ and $Y^{\prime}(k)$ :

$$
\widehat{P S}(1) \approx \frac{1}{4} \arg \left[\frac{1}{N} \sum_{i=1}^{N} X^{\prime *}(i)^{4} X^{\prime}(i-1)^{4}\right],
$$

where $\boldsymbol{N}$ is the number of samples used in the estimation process.

- The FFT algorithm estimates $\Delta f$ as the maximum value of the Fourier transform modulus of the equalized sequence raised to the fourth power:

$$
\widehat{\Delta f} \approx \frac{\max _{f}\left[\left|F F T\left(X^{\prime 4}\right)\right|\right]}{4} .
$$

\section{EXPERIMENTAL RESULTS FROM OFFLINE POST-PROCESSING}

Figure 6 shows the experimental setup for the $112 \mathrm{~Gb} / \mathrm{s}$ DP-QPSK system. At the transmitter, a pseudorandom binary sequence (PRBS) is generated at $28 \mathrm{~Gb} / \mathrm{s}$. The original sequence drives the inphase component of an I/Q Mach-Zehnder modulator (nested structure), while a 51-bits delayed 
version drives the quadrature component. The resulting optical signal is subsequently RZ-modulated by a pulse carver. Lastly, the polarization multiplexed signal is generated by splitting the optical signal into two orthogonal polarization orientations (using a polarization beam splitter (PBS)), decorrelating the vertical and horizontal components by a 2 meters polarization maintaining fiber (PMF), and recombining the two components in a polarization beam combiner (PBC).

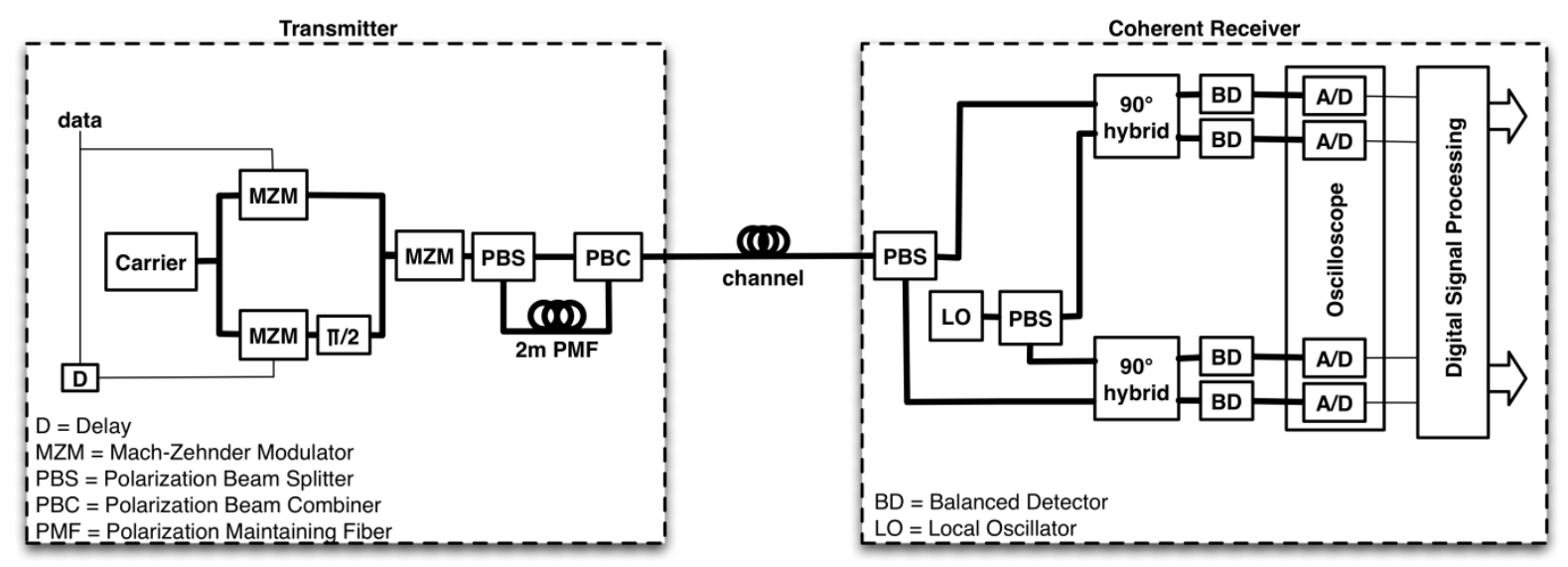

Fig. 6. Experimental setup.

The receiver is composed of the traditional polarization diversity front-end architecture followed by

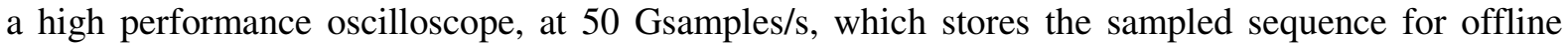
post-processing. All investigated cases used the single-spike initialization for the butterfly filters. BER values were obtained by comparing the decoded sequence with 550,000 transmitted symbols. The initial 10,000 training symbols were not used for BER computation.
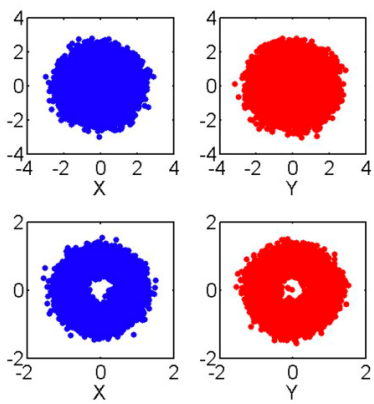

(a) OSNR $=17 \mathrm{~dB}$
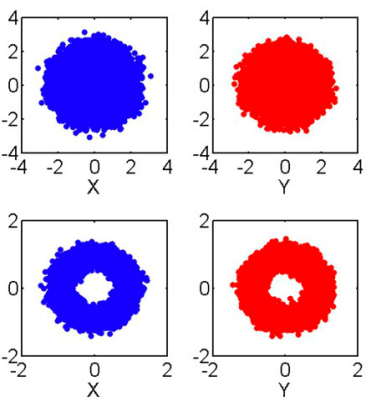

(d) OSNR $=20 \mathrm{~dB}$
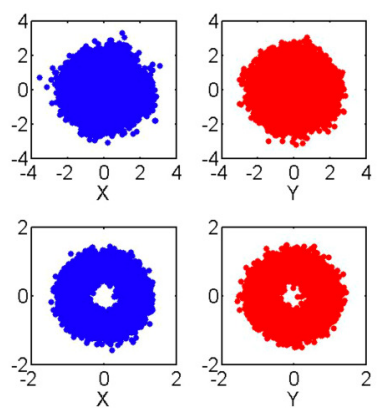

(b) OSNR $=18 \mathrm{~dB}$
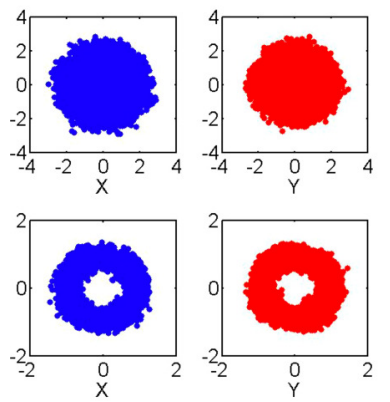

(e) OSNR $=21 \mathrm{~dB}$
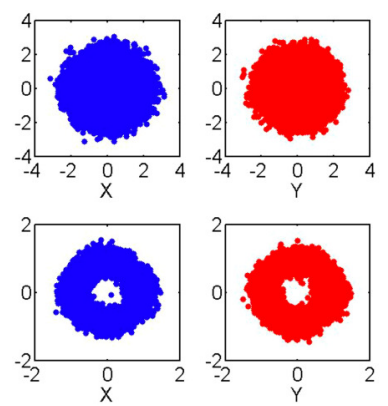

(c) OSNR $=19 \mathrm{~dB}$
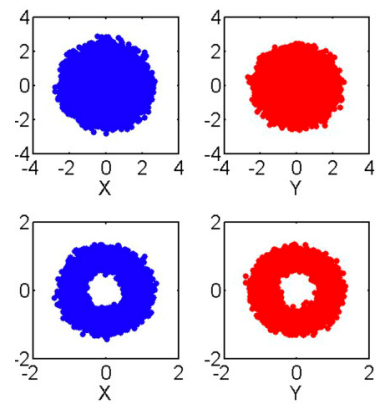

(f) OSNR $=22 \mathrm{~dB}$

Fig. 7. Constellations of the received and CMA-demultiplexed signals for the investigated OSNRs after normalization (10,000 symbols). The circle-like upper curves indicate a strong mixture of the vertical and horizontal polarization orientations. 
The first investigated scenario consists of a fiber-free setup with a noise-loading amplifier. In Fig. 7 , the circle-like constellations of the received signals after normalization indicate a strong mixture of the vertical and horizontal polarization orientations for the investigated OSNRs (at $0.1 \mathrm{~nm}$ ). We analyze the performance of the two frequency recovery algorithms.

Figure 8 shows the estimated frequency offset $\Delta f$ as a function of the system OSNR, computed using all $X^{\prime}$ and $Y^{\prime}$ samples obtained after the training phase. Since the frequency offset is in the order of some few dozens of $\mathrm{MHz}$, its measurement would require very high resolution optical spectral analyzers. Alternatively, we analyze the dispersion of the four estimated values: the phase-difference algorithm using $X^{\prime}$ and $Y^{\prime}$, and the FFT algorithm using $X^{\prime}$ and $Y^{\prime}$. At high OSNR, the estimates produced by the two algorithms coincide, however, as the OSNR decreases, the estimates of the phase-difference algorithm diverge. This indicates that, although the phase-difference algorithm is less computationally complex, it is also more susceptible to additive noise.

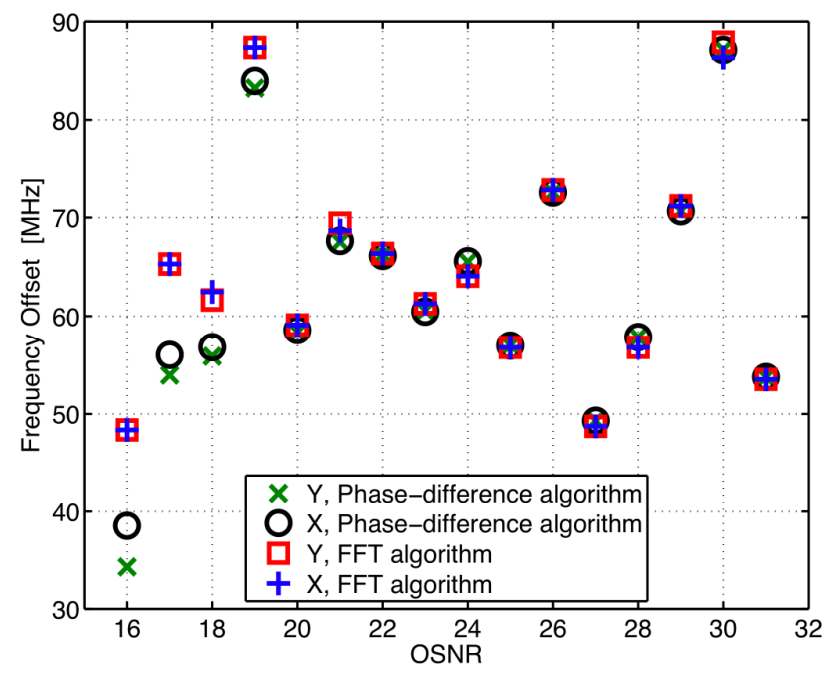

Fig. 8. Performance of the phase-difference and FFT frequency recovery algorithms.

Figure 9 shows the BER versus OSNR curves for the two investigated systems (CMA or DD-LMS filter tracking) for the butterfly equalizer with $5 \mathrm{~T} / 2$-spaced taps and frequency recovery using the phase-difference algorithm. Both systems achieved error-free transmission for OSNR values higher than $22 \mathrm{~dB}$, and the CMA exhibited a slightly lower BER than the DD-LMS algorithm for low OSNR values. This may be explained by the fact that the DD-LMS algorithm depends on correct decisions, and symbol errors degrade the overall DD equalizer performance.

In general CMA equalization is preferred because of its immunity to phase noise and residual frequency offset. The performance of the equalization process, for a given residual frequency offset (in OSNR of $19 \mathrm{~dB}$ ), can be observed in Fig. 10. Even though CMA has the advantage of being immune to the residual frequency offset, the carrier recovery process is still affected. As expected, the DD-LMS algorithm exhibited a lower tolerance to the residual frequency offset, because the equalization and the carrier recovery processes are interdependent. 


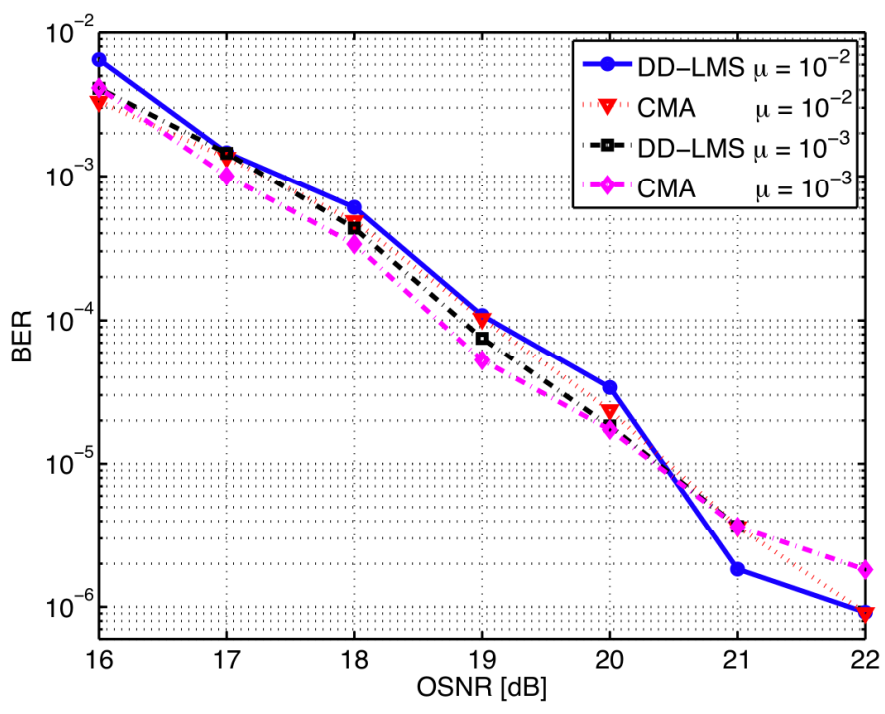

Fig. 9. Performance curves for the CMA and DD-LMS systems under amplified spontaneous emission noise.

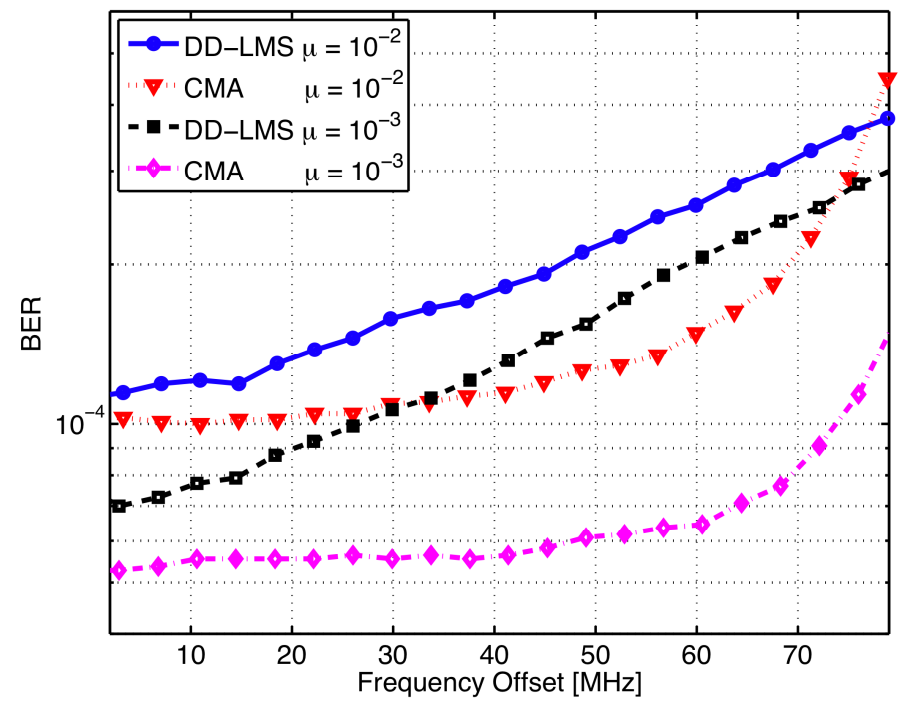

Fig. 10. Performance curves for the CMA and DD-LMS systems under amplified spontaneous emission noise and residual frequency offset.

In the second investigated scenario, we post-processed signals impaired by residual chromatic dispersion $(850 \mathrm{ps} / \mathrm{nm})$ and polarization mode dispersion (50 ps DGD), at OSNR = 18 dB. Figure 11 shows the BER as a function of the number of adaptive filter taps. The dot-dashed and solid curves correspond to the CMA and DD-LMS equalizers, respectively, when the bulk dispersion compensating filter is designed for $850 \mathrm{ps} / \mathrm{nm}$. The performance of the fully-adaptive CMA equalizer is indicated by the dotted line. The fully-adaptive DD-LMS algorithm, in turn, exhibited an unsatisfactory performance, and was therefore omitted from the diagram. Clearly, the bulk dispersion compensator allowed to reduce the required number of adaptive filter taps, however, a minimum number of filter taps was still needed to equalize the PMD. Note that the fully-adaptive CMA equalizer achieved the lowest BER values after 13 taps. This inferior performance of the investigated bulk dispersion compensator may be attributed to amplitude response ripple evidenced in Fig. 2. 


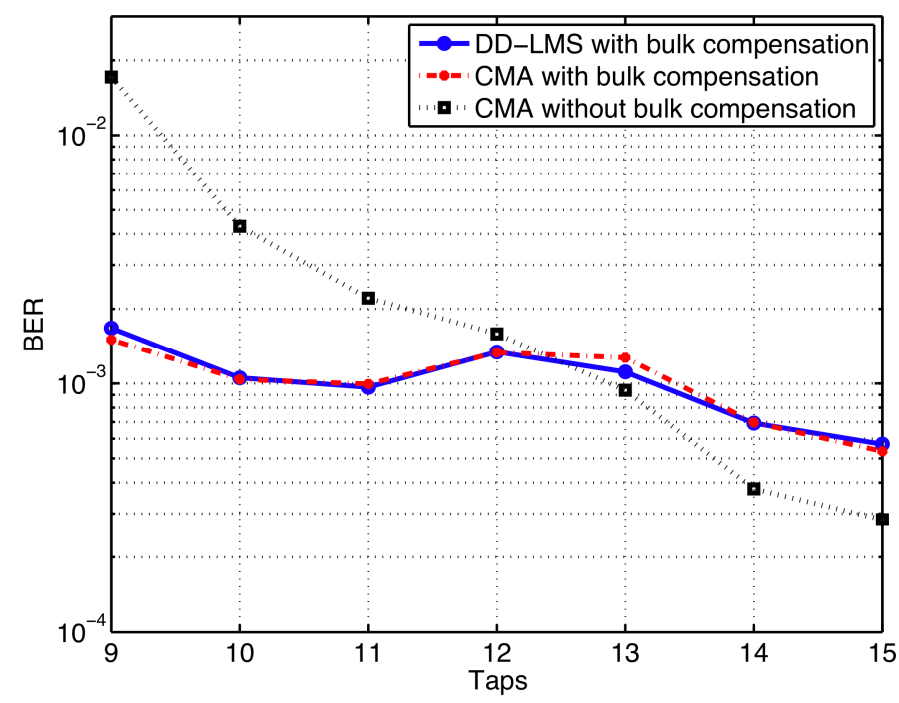

Fig. 11. Performance curves for Systems 1 and 2 under amplified spontaneous emission noise, first-order PMD (DGD $=50 \mathrm{ps})$ and residual $\mathrm{CD}=850 \mathrm{ps} / \mathrm{nm}$.

\section{CONCLUSION}

We analyzed the performance of several signal processing algorithms for optical DP-QPSK 112 $\mathrm{Gb} / \mathrm{s}$ coherent receivers using the offline post-processing of experimental data. In particular, two systems based on CMA and DD-LMS equalization were investigated. Both systems performed similarly in the separation of polarization multiplexed data, provided that a possible frequency offset between transmitter and local oscillator lasers was properly compensated. Two frequency recovery algorithms were compared, and the FFT-based alternative exhibited a higher tolerance to additive noise, though being more computationally complex. We also investigated the performance of the two equalization strategies combined with bulk dispersion compensators, in the presence of chromatic dispersion and the first order polarization mode dispersion (DGD). The results indicated that timedomain equalizer in some cases impairs the BER due to ripple in its amplitude response, which may account for some noise amplification.

\section{ACKNOWLEDGMENT}

This work was supported by the Innovation Center, Ericsson Telecomunicações S.A., Brazil.

\section{REFERENCES}

[1] Fatadin, S. Savory, and D. Ives, "Compensation of quadrature imbalance in an optical QPSK coherent receiver," Photonics Technology Letters, IEEE, vol. 20, no. 20, pp. 1733 -1735, oct.15, 2008.

[2] S. J. Savory, "Digital filters for coherent optical receivers," Opt. Express, vol. 16, no. 2, pp. 804-817, 2008. [Online]. Available: http://www.opticsexpress.org/abstract.cfm?URI=oe-16-2-804

[3] S. J. Savory, G. Gavioli, R. I. Killey, and P. Bayvel, "Electronic compensation of chromatic dispersion using a digital coherent receiver," Opt. Express, vol. 15, no. 5, pp. 2120-2126, 2007. [Online]. Available: http://www.opticsexpress.org/abstract.cfm?URI=oe-15-5-2120

[4] S. Haykin, "Unsupervised adaptive filtering," vol. 2, no. 5, 2000. 
[5] J. Johnson, R., P. Schniter, T. Endres, J. Behm, D. Brown, and R. Casas, "Blind equalization using the constant modulus criterion: a review," Proceedings of the IEEE, vol. 86, no. 10, pp. 1927-1950, Oct 1998.

[6] F. A. Garcia, D. A. Mello, and H. Waldman, "Feedforward carrier recovery for polarization demultiplexed signals with unequal signal to noise ratios," Opt. Express, vol. 17, no. 10, pp. 7958-7969, 2009. [Online]. Available: http://www.opticsexpress.org/abstract.cfm?URI=oe-17-10-7958

[7] E. Ip, J. M. Kahn, "Feedforward carrier recovery for coherent optical communications," J. Lightwave Technol., vol. 25, pp. 2675-2692, 2007.

[8] A. Vgenis, C. S. Petrou, C. B. Papadias, I. Roudas, and L. Raptis, "Nonsingular constant modulus equalizer for PDM-QPSK coherent optical receivers,” Photonics Technology Letters, IEEE, vol. 22, pp. 45-47, 2010. 